\title{
Modelling and Validation of Hybrid Heavy Duty Vehicles with Exhaust Aftertreatment Systems
}

\author{
Olov Holmer Lars Eriksson \\ Vehicular Systems, Dept. Electrical Engineering, Linköping University, Sweden, \{ olov.holmer, \\ lars.eriksson\} @liu.se
}

\begin{abstract}
A model of a hybrid electric vehicle including an aftertreatment system is developed and validated. The model describes a vehicle with the same parallel hybrid architecture that is commonly used in commercial heavy duty vehicles and is validated using data gathered from vehicles during real world driving. The goal with the model is to describe the main dynamics of the system and give accurate estimations of fuel consumption and emissions while at the same time keeping simulation times short. The model consists of several sub components, out of which the most important ones are: combustion engine, electric motor, aftertreatment system, driveline, and vehicle chassis. The different components are interchangeable making it possible for the user to change specific components to make the model fit their needs.
\end{abstract}

Keywords: hybrid heavy duty vehicle, aftertreatment system, vehicle model

\section{Introduction}

When designing controls systems, models of the system often makes the work significantly easier. This is because the development can be done using simulations which often is faster, and cheaper than using the real system. To facilitate model based development there is a need to have simulation models for the system, and development of models is therefore important. Well documented models for conventional and hybrid heavy duty vehicles exist. Some models of hybrid vehicles with aftertreatment systems also exist, like (Willems and Foster, 2009), however, to the authors knowledge no work where the interplay between exhaust system and hybrid vehicle, where the engine can be shut of, is studied and modeled can be found. Therefore we here aim to fill this gap.

\subsection{Contributions}

The main contribution is a complete vehicle model with powertrain, vehicle chassis, and after treatment system. In addition, some new component models have been created and a study where the interplay between the aftertreatment system and the hybrid vehicle has been done, to make sure the model can handle all conditions that arise in a hybrid vehicle where the engine can be shut of.

\section{Modeling}

The hybrid architecture used in the model is a parallel configuration where the engine and motor is connected before the gearbox using a torque coupler. The gearbox is then used to connect the torque coupler with the wheels. In Figure 1 the vehicle configuration and the different components in the model are shown.

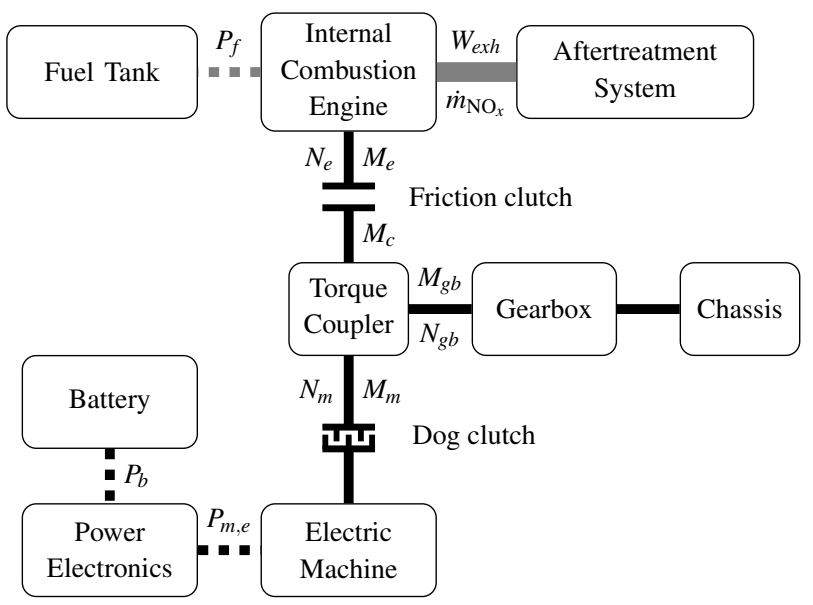

Figure 1. Configuration of the hybrid vehicle.

The sub-models are taken from previous work. However, some work have been done to make the models work in a hybrid vehicle application where the engine is sometimes turned of and the mass flow through the engine and aftertreatment system is zero. Also, a few new models have been developed that describe effects that are negligible in conventional vehicles but become clear in hybrid vehicles. These effects mainly come from that a hybrid vehicle can shut of the engine resulting in zero mass flow through the engine and aftertreatment system. In this section the sub-models are presented.

\subsection{Engine}

The engine model is taken from (Wahlström and Eriksson, 2011). Some modifications have been done to the model so that it is able to simulate zero exhaust mass flow when the engine is turned of and the model has also been extended with models for a compression release brake, an exhaust brake (or back pressure valve), and models for the composition of the exhaust gas. The model is complex 
and well explained in (Wahlström and Eriksson, 2011), therefore only the essential equations and the modifications made to the model are presented here.

The remainder of this section is organized as follows: first the states in the model is described, then the model of the compression release brake is presented followed by an explanation of how the model has been modified to allow zero mas flow, and finally some of the most important equations in the model are presented.

\subsubsection{States}

The model has 9 states, out of these, four describe the main dynamics of the system and they are pressure in the intake manifold, $p_{i m}$, pressure in the exhaust manifold, $p_{e m}$, pressure before the back pressure valve, $p_{b p v}$, and turbo speed, $\omega_{t}$. Two states describe oxygen mass fraction, one in the intake manifold, $X_{O_{i m}}$, and one in the exhaust manifold, $X_{O_{i m}}$. The last three describe dynamics in the actuators, $\tilde{u}_{e g r, 1}$ and $\tilde{u}_{e g r, 2}$ describes the dynamics of the EGR valve and $\tilde{u}_{v g t}$ describes the dynamics of the VGT.

The differential equations for the manifold pressures are based on isothermal models (Eriksson and Nielsen, 2014), which gives

$$
\begin{gathered}
\dot{p}_{i m}=\frac{R_{a} T_{i m}}{V_{i} m}\left(W_{c}+W_{e g r}-W_{e i}\right) \\
\dot{p}_{e m}=\frac{R_{a} T_{e m}}{V_{e} m}\left(W_{e o}-W_{t}-W_{e g r}\right) \\
\dot{p}_{b p v}=\frac{R_{a} T_{a f t . t r u b}}{V_{b p v}}\left(W_{t}-W_{b p v}\right)
\end{gathered}
$$

where $W_{i}, i \in\{c, e g r, e i, e o, b p v\}$ is the mass flows in and out of the volumes. $W_{c}$ is the compressor mass flow which mainly depends on $\omega_{t}$ and $p_{i m}$. $W_{\text {egr }}$ is the exhaust gas recirculation mass flow which mainly depends on the ratio $p_{i m} / p_{e m}$ and the control signal $u_{e g r} . w_{e i}$ is the cylinder-in mass flow which mainly depends on $p_{i m}$ and $n_{e}, W_{e o}$ is the engine out mass flow which is the sum of $W_{e i}$ and the injected amount of fuel. $W_{b p v}$ is the mass flow past the back pressure valve which is explained below.

The dynamics of the turbo speed follows Newton's second law

$$
\dot{\omega}_{t}=\frac{P_{t} \eta_{m}-P_{c}}{J_{t} \omega_{t}}
$$

where $P_{t}$ is the power delivered by the turbine, $P_{c}$ is the power required by the compressor, $J_{m}$ is the turbo inertia, and $\eta_{m}$ is the mechanical efficiency of the turbocharger. $P_{t}$ mainly depends on the ratio $p_{e m} / p_{b p v}, T_{e m}$, and the control signal $u_{v g t} . P_{c}$ mainly depends on the ratio $p_{i m} / p_{a m b}$.

The differential equations for the oxygen mass fractions

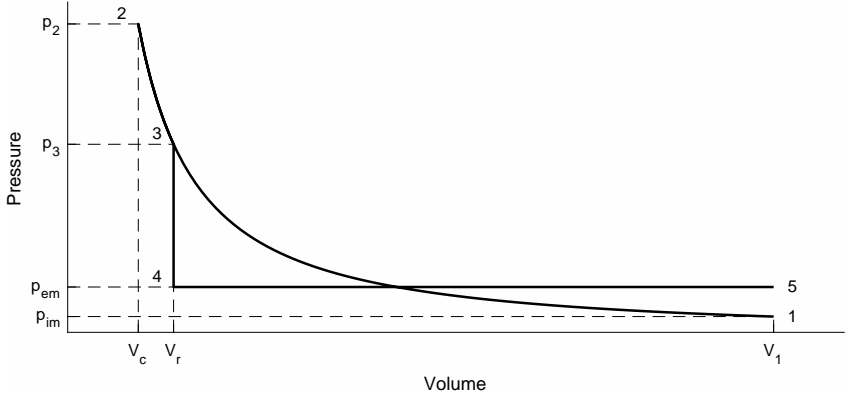

Figure 2. p-V diagram for the compression release brake.

are

$$
\begin{array}{r}
\dot{X}_{O_{i m}}=\frac{R_{a} T_{i m}}{p_{i m} V_{i m}}\left(\left(X_{O_{e m}}-X_{O_{i m}}\right) W_{e g r}\right. \\
\left.\quad+\left(X_{O_{c}}-X_{O_{i m}}\right) W_{e o}\right) \\
\dot{X}_{O_{e m}}=\frac{R_{e} T_{e m}}{p_{e m} V_{e m}}\left(X_{O_{e}}-X_{O_{e m}}\right) W_{e o}
\end{array}
$$

where $X_{O_{c}}=23.14 \%$ is the oxygen concentration of air passing through the compressor and $X_{O_{e}}$ is the oxygen concentration of the exhaust gases coming from the engine.

The states $\tilde{u}_{e g r, 1}$ and $\tilde{u}_{e g r, 2}$ are both first order systems with $u_{e g r}$ as input and together they make up the dynamics of the EGR valve. The state $\tilde{u}_{v g t}$ is a first order system with $u_{v g t}$ as input and describe the dynamics of the VGT.

\subsubsection{Compression release brake}

To model the compression release brake we study an ideal cycle shown in Figure 2. The cycle is without combustion and a compression release is done after top dead center at a volume $v_{r} \geq v_{c}$.

\section{Exhaust Temperature}

To get the exhaust temperature, when using the compression release brake, we start by calculating temperature at the different steps in the cycle.

\section{Compression (1-2)}

$$
T_{2}=T_{1}\left(\frac{v_{1}}{v_{2}}\right)^{\gamma-1}=r_{c}^{\gamma-1}
$$

\section{Expansion (2-3)}

$$
\begin{aligned}
T_{3}=T_{1}\left(\frac{v_{2}}{v_{3}}\right)^{\gamma-1}=p_{1}\left(\frac{v_{1}}{v_{2}}\right)^{\gamma-1} & \left(\frac{v_{2}}{v_{3}}\right)^{\gamma-1} \\
= & T_{1}\left(\frac{v_{1}}{v_{3}}\right)^{\gamma-1}
\end{aligned}
$$

\section{Compression release (3-4)}

$$
T_{4}=T_{3}=T_{e x h}
$$


The exhaust temperature is thus

$$
T_{e x h}=T_{1}\left(\frac{v_{1}}{v_{3}}\right)^{\gamma-1}
$$

as can be seen it depends on the ration between $v_{1}$ and $v_{3}$, we therefore introduce

$$
r_{r}=\frac{v_{1}}{v_{3}} \in\left[1, r_{c}\right]
$$

as a tuning parameter.

\section{Brake Torque}

To calculate the brake torque we first calculate the work done during this part of the cycle

$$
\begin{gathered}
W_{c r b}=\int_{1-5}\left(p-p_{a m b}\right) d v=\int_{v_{1}}^{v_{2}} p d v+\int_{v_{2}}^{v_{3}} p d v \\
+\int_{v_{3}}^{v_{4}} p d v+\int_{v_{4}}^{v_{5}} p d v-\int_{1-5} p_{a m b} d v \\
=\int_{v_{1}}^{v_{3}} p_{1}\left(\frac{v_{1}}{v}\right)^{\gamma} d v+\int_{v_{4}}^{v_{5}} p_{e m} d v \\
=\left[p_{1} \frac{v_{1}^{\gamma}}{(1-\gamma) v^{\gamma-1}}\right]_{v_{1}}^{v_{3}}+\left(v_{5}-v_{4}\right) p_{e m} \\
=\frac{p_{1} v_{1}}{1-\gamma}\left(\left(\frac{v_{1}}{v_{3}}\right)^{\gamma-1}-1\right)+\left(v_{5}-v_{4}\right) p_{e m} \\
=\frac{p_{1} v_{1}}{1-\gamma}\left(r_{r}^{\gamma-1}-1\right)+\left(v_{5}-v_{4}\right) p_{e m}
\end{gathered}
$$

this term can then be added to the torque model.

\subsubsection{Engine Shutdown and Zero Mass Flow}

Since the engine model contains some singularities at zero mas flow the original model has a saturation on the engine speed that ensures that the mass flow does not get to low. This saturation has been set at 400 RPM, which is well below the normal working range of the engine, however, in a hybrid, where the engine can be shut down, this causes problems since the engine then continues to blow cold air through the aftertreatment system.

To remedy this a switching function is used to calculate the exhaust mass flow, $W_{\text {exh }}$. The switching function chooses between the turbine mass flow, $W_{t}$, from the original model and a mass flow calculated based on the volumetric efficiency, $\eta_{v o l}\left(p_{i m}, N_{e}\right)$, of the engine in the following way

$$
W_{\text {exh }}= \begin{cases}W_{t}, & N_{e} \geq 400 \\ \frac{\eta_{v o l}\left(p_{i m}, N_{e}\right) p_{i m} N_{e} V_{d}}{120 R_{a} T_{i m}}, & N_{e}<400\end{cases}
$$

The result of the switching function can be seen in Figure 3. In the figure the engine is first idling at around 500 RPM and after one second the engine is turned of. As can be seen the exhaust mass flow in the original model first decreases but when the engine speed reaches 400 RPM the exhaust mas stops falling and remains constant, for the extended model however the exhaust mass flow continues to drop all the way to zero. It should also be noted that the switching between the two models is smooth.
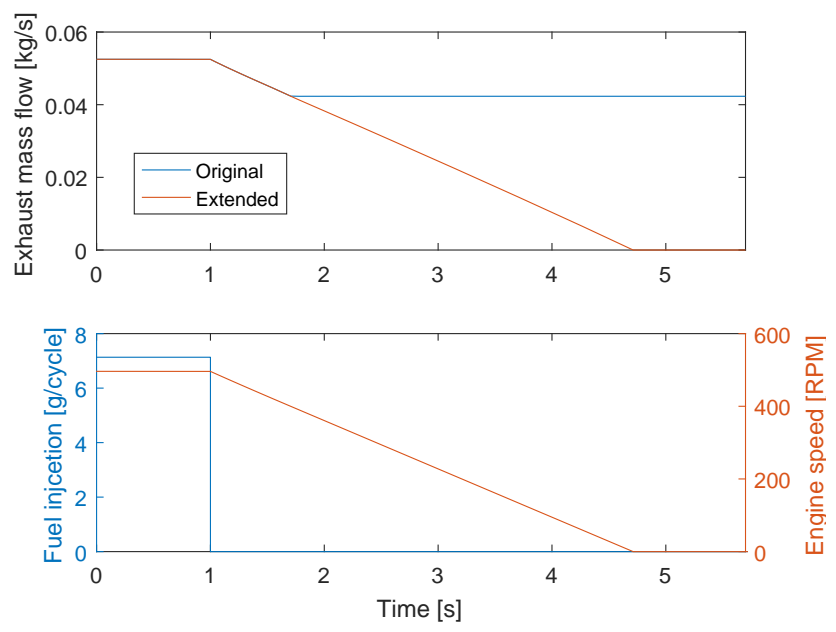

Figure 3. Figure showing mass flow and engine speed during an engine shutdown from both the original, saturated, model and the extended motel.

\subsubsection{Equations}

Here the equations for the engine torque, turbine mass flow, back pressure valve, exhaust temperature, and exhaust gas conditions are presented.

\section{Engine torque}

The engine torque, $M_{e}$, is modeled using four components: gross indicated torque, $M_{i g}$, pumping torque, $M_{p}$, friction torque $M_{\text {fric }}$, and brake torque form the compression release brake, $M_{c r b}$, in the following way

$$
M_{e}=M_{i g}-M_{p}-M_{f r i c}+M_{c r b} .
$$

The pumping work is calculated as

$$
M_{p}=\frac{V_{d}}{4 \pi}\left(p_{e m}-p_{i m}\right),
$$

the gross indicated torque is calculated according to

$$
M_{i g}=\frac{u_{\delta} n_{c y l} q_{H V} \eta_{i g c h}\left(1-\frac{1}{r_{c} c_{c y l}^{-1}}\right)}{4 \pi}
$$

the friction torque is calculated using

$$
M_{f r i c}=\frac{V_{d}}{4 \pi}\left(c_{f r i c, 1}\left(\frac{n_{e}}{100}\right)^{2}+c_{f r i c, 2} \frac{n_{e}}{100}+c_{f r i c, 3}\right),
$$

and the brake torque from the compression release brake is calculated as described in Section 2.1.2. 


\section{Turbine mass flow}

The turbine mass flow is modeled as

$$
W_{t}=\frac{A_{v g t, \max } P_{e m} f_{\pi_{t}}\left(\pi_{t}\right) f_{v g t}\left(\tilde{u}_{v g t}\right)}{\sqrt{T_{e m} R_{e}}}
$$

where $f_{\pi_{t}}$ and $f_{v g t}$ are functions defined in (Wahlström and Eriksson, 2011). However, due to singularities in the model the engine and turbine speed have lover limit which implicitly induces a lower limit on $W_{t}$. Therefore, at engine speeds lower than this limit the turbine mass flow is instead taken as the mass flow given by the speed and volumetric efficiency of the engine:

$$
W_{t}=\frac{\eta_{\text {vol }} p_{i m} n_{e} V_{d}}{120 R_{a} T_{i m}}
$$

where the volumetric efficiency, $\eta_{v o l}$, is modeled as

$$
\eta_{v o l}=c_{v o l, 1} \sqrt{p_{i m}}+c_{v o l, 2} \sqrt{n_{e}}+c_{v o l, 3} .
$$

Back pressure valve To model the back pressure valve a control volume with volume, $V_{b p v}$, and pressure, $p_{b p v}$, is first added after the turbine. The inflow to this control volume is the turbine mass flow and the out flow is the flow past the back pressure valve. The flow past the back pressure valve is modeled using a throttle mass flow model (Eriksson and Nielsen, 2014)

$$
W_{b p v}=\frac{p_{u s}}{\sqrt{R T u s}} A_{b p v} \Psi_{l i}(\Pi)
$$

where

$$
\Pi=\max \left(\frac{p_{\text {bpv }}}{p_{\text {eats }}},\left(\frac{2}{\gamma+1}\right)^{\frac{\gamma}{\gamma-1}}\right)
$$

and

$$
\Psi_{l i}(\Pi)=\left\{\begin{array}{ll}
\sqrt{\frac{2 \gamma}{\gamma-1}\left(\Pi^{\frac{2}{\gamma}}-\Pi^{\frac{\gamma+1}{\gamma}}\right)}, & \Pi \leq \Pi_{l i} \\
\sqrt{\frac{2 \gamma}{\gamma-1}\left(\Pi_{l i}^{\frac{2}{\gamma}}-\Pi_{l i}^{\frac{\gamma+1}{\gamma}}\right)} \frac{1-\Pi}{1-\Pi_{l i}}, & \Pi>\Pi_{l i}
\end{array} .\right.
$$

The linear region, $\Pi>\Pi_{l i}$, is used to overcome problems when simulating the system that comes from that the $\Psi$ does not fulfill the Lipschitz condition when the pressure ratio is equal to one (Eriksson and Nielsen, 2014).

\section{Exhaust temperature}

The cylinder-out temperature, $T_{e}$, is modeled using calculations for an ideal Seliger cycle and is explained in (Wahlström and Eriksson, 2011) when the compression release brake is not used. When the compression release brake is used $T_{e}$ is modeled as described in Section 2.1.2. Between the turbine and cylinder heat losses are modeled so that the temperature before the turbine, $T_{e m}$, is colder than the cylinder-out temperature.

The temperature after the turbine is calculated using the turbine efficiency, $\eta_{t}$, defined in (Heywood, 1988), giving

$$
T_{a f t . t u r b .}=T_{e m}\left(1-\eta_{t}\left(1-\Pi_{t}^{1-1 / \gamma_{e}}\right)\right)
$$

The temperature of the pipe between the engine and the EATS is governed by the following dynamic model (Eriksson, 2002)

$$
\dot{T}_{w}=\dot{Q}_{i}\left(T_{w}, T_{a f t . t u r b .}\right)-\dot{Q}_{e}\left(T_{w}, T_{a m b}\right)
$$

where

$$
\begin{gathered}
\dot{Q}_{i}=h_{g, i} A\left(T_{a f t . t u r b .}-T_{w}\right), \\
h_{g, i}=\frac{1-e^{-\frac{h_{c v, i} A}{W c p}}}{\frac{h_{c v, i} A}{W c_{p}}} h_{c v, i}
\end{gathered}
$$

and

$$
\dot{Q}_{e}=A\left(h_{c v, e}\left(T_{w}-T_{a m b}\right)+F_{v} \varepsilon \sigma\left(T_{w}^{4}-T_{a m b}^{4}\right)\right) .
$$

The temperature of the gas entering the EATS can now be calculated as

$$
T_{E A T S}=T_{w}+\left(T_{a f t . t u r b .}-T_{w}\right) e^{-\frac{h_{c v, i} A}{W c p}}
$$

\section{Exhaust gas conditions}

The $\mathrm{NO}_{\mathrm{x}}, \mathrm{NO}_{\mathrm{x}}$, and $\mathrm{O}_{2}$ concentrations are calculated based on maps depending on the engine torque and speed. The rest of the values are taken direct as the values given by the dynamic engine model.

\subsection{Electrical Components}

Here the models of the electrical components in the powertrain are described.

\subsubsection{Battery}

The battery model is based on a Thévelin equivalence circuit with an open circuit voltage, $U_{o c}$, that depends on the state of charge, $S O C$, and internal resistance $R_{i}$.

The battery current, $I_{b}$, is calculated as

$$
I_{b}=\frac{U_{o c}(S O C)}{2 R_{i}}-\sqrt{\frac{U_{o c}(S O C)^{2}}{4 R_{i}^{2}}-\frac{P_{b}}{R_{i}}} .
$$

where $P_{b}$ is the terminal power of the battery, $R_{i}$ is the internal resistance of the battery, and $U_{o c}$ is the open circuit voltage. The dynamics of the $S O C$ is

$$
S \dot{O} C=-\frac{I_{b}}{Q_{0}}
$$

and $U_{o c}$ is calculated using maps based on the $S O C$. 


\subsubsection{Power Electronics}

The model of the power electronics takes a desired motor power, $P_{m, d}$, and the required power from the auxiliary units, $P_{a u x}$, and calculates the necessary battery power, $P_{b}$, and actual electrical power to the motor $P_{m, e}$. Since the losses in the power electronics are included in the motor model, the power electronics is modeled as ideal. This means that the model of the power electronics only have to ensure that the battery and electric machine work within there limits. The battery power is calculated as

$$
P_{b}= \begin{cases}P_{b, \max }, & P_{m, d}+P_{a u x} \geq P_{b, \max } \\ P_{m, d}+P_{\text {aux }}, & P_{b, \min } \leq P_{m, d}+P_{a u x} \leq P_{b, \max } \\ P_{b, \min }, & P_{m, d}+P_{\text {aux }} \leq P_{b, \min }\end{cases}
$$

and the motor power is calculated as

$$
P_{m, e}=\left\{\begin{array}{l}
P_{b, \text { max }}-\bar{P}_{a u x}, \quad P_{m, d} \geq P_{b, \max }-\bar{P}_{a u x} \\
P_{m, d}, P_{b, \min }-\bar{P}_{a u x} \leq P_{m, d} \leq P_{b, \max }-\bar{P}_{a u x} \\
P_{b, \min }-\bar{P}_{a u x}, \quad P_{m, d} \leq P_{b, \min }-\bar{P}_{a u x}
\end{array}\right.
$$

where

$$
\bar{P}_{\text {aux }}= \begin{cases}P_{b, \max }, & P_{\text {aux }} \geq P_{b, \max } \\ P_{\text {aux }}, & P_{b, \min } \leq P_{\text {aux }} \leq P_{b, \max } \\ P_{b, \min }, & P_{\text {aux }} \leq P_{b, \min }\end{cases}
$$

\subsubsection{Electric Motor}

The motor model is a static model of a permanent magnet synchronous machine taken from (Sundström et al., 2015). When the model is parameterized the losses in the power electronics are included and in that way the model also include these losses. The current in the stator, $I_{m}$, is calculated as

$$
I_{m}=\frac{1}{R_{m}}\left(U_{m}-k_{i} \omega_{m}\right)
$$

where, $U_{m}$, is the voltage over the motor, calculated as

$$
U_{m}=\frac{k_{i} \omega_{m}}{2}+\sqrt{\frac{k_{i}^{2} \omega_{m}^{2}}{4}+P_{m, e} R_{m}} .
$$

The output torque is calculated as

$$
M_{m}=k_{a} I_{m}-c_{f} \omega_{m}
$$

where $k_{a}$ is defined as

$$
k_{a}=\left\{\begin{array}{ll}
k_{i} \eta_{m}, & I_{m} \geq 0 \\
\frac{k_{i}}{\eta_{m}}, & I_{m}<0
\end{array} .\right.
$$

The limits $P_{m, \max }$ and $P_{m, \min }$ are calculated using maps depending on $n_{m}$.

\subsection{Aftertreatment system}

The aftertreatment system consists of three active components: a diesel oxidation catalyst, DOC, a diesel particulate filter, DPF, and a selective catalytic reduction, SCR, catalyst. The components are also enclosed inside a silencer.

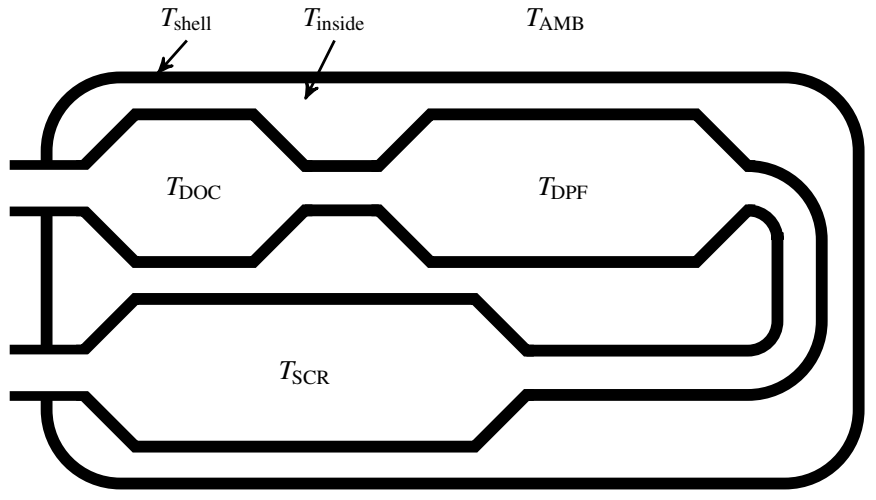

Figure 4. Temperatures of the different components inside the silencer.

\subsubsection{Silencer}

The silencer model has one state for the temperature inside the silencer, $T_{\text {inside }}$, governed by the following differential equation

$$
\begin{array}{r}
\dot{T}_{\text {inside }}=\frac{h_{\text {solid } \leftrightarrow \text { air }}}{c_{p, \text { air }} m_{\text {air }}}\left(A_{\text {doc }}\left(T_{\text {doc }}-T_{\text {inside }}\right)\right. \\
+A_{\text {dpf }}\left(T_{\text {dpf }}-T_{\text {inside }}\right)+A_{\text {scr }}\left(T_{\text {scr }}-T_{\text {inside }}\right) \\
\left.+A_{\text {shell }}\left(T_{\text {shell }}-T_{\text {inside }}\right)\right)
\end{array}
$$

and one state for the temperature of the shell of the silencer, $T_{\text {shell }}$, governed by the following differential equation

$$
\begin{array}{r}
\dot{T}_{\text {shell }}=\frac{A_{\text {shell }}}{c_{p, \text { air }} m_{\text {air }}}\left(h_{\text {solid } \leftrightarrow \text { air }}\left(T_{\text {inside }}-T_{\text {shell }}\right)\right. \\
\left.+h_{\text {solid } \leftrightarrow \text { amb }}\left(T_{\text {amb }}-T_{\text {shell }}\right)\right)
\end{array}
$$

\subsubsection{Energy Balance Modeling}

To model the temperature inside the substrates the models in (Winkler et al., 2003; Van Helden et al., 2004) is used as a starting point. The following energy balance is used in the gas phase

$$
\varepsilon \rho_{g} C_{p, g} \frac{d T_{g}}{d t}=-v \varepsilon \rho_{g} C_{p, g} \frac{\partial T_{g}}{\partial x}-h_{g \leftrightarrow s} a_{g \leftrightarrow s}\left(T_{g}-T_{s}\right)
$$

and in the solid phase

$$
\begin{array}{r}
(1-\varepsilon) \rho_{s} C_{p, s} \frac{d T_{s}}{d t}=(1-\varepsilon) \lambda_{s} \frac{\partial^{2} T_{s}}{\partial x^{2}}+h_{g \leftrightarrow s} a_{g \leftrightarrow s}\left(T_{g}-T_{s}\right) \\
-h_{s \leftrightarrow a} a_{s \leftrightarrow a}\left(T_{s}-T_{a}\right)+\sum_{\text {reactions }} r_{j} \Delta H_{j}
\end{array}
$$

To simplify the model the following assumptions are then made

1. Instantaneous equilibrium between brick material and exhaust gas, resulting in $d T_{g} / d t=0$ and $T_{g}=T_{s}$

2. Conductive heat transport $<<$ convective heat transport, and the term $(1-\varepsilon) \lambda_{s} \frac{\partial^{2} T_{s}}{\partial x^{2}}$ in (42) can therefore be omitted 
3. No significant endothermic or exothermic reactions, i.e. we assume

$\sum_{\text {reactions }} r_{j} \Delta H_{j}=0$

Using the assumption $d T_{g} / d t=0$ and (41), we get

$$
v \varepsilon \rho_{g} C_{p, g} \frac{\partial T_{g}}{\partial x}=-h_{g \leftrightarrow s} a_{g \leftrightarrow s}\left(T_{g}-T_{s}\right)
$$

Using assumptions 1 and 2, (42) becomes

$$
\begin{aligned}
(1-\varepsilon) \rho_{s} C_{p, s} \frac{d T_{s}}{d t}=h_{g \leftrightarrow s} a_{g \leftrightarrow s}( & \left.T_{g}-T_{s}\right) \\
& -h_{s \leftrightarrow a} a_{s \leftrightarrow a}\left(T_{s}-T_{a}\right)
\end{aligned}
$$

By combining (43), (44) and the assumption that $T_{g}=T_{S}$ we get

$$
(1-\varepsilon) \rho_{s} C_{p, s} \frac{d T_{g}}{d t}=-v \varepsilon \rho_{g} C_{p, g} \frac{\partial T_{g}}{\partial x}-h_{s \leftrightarrow a} a_{s \leftrightarrow a}\left(T_{g}-T_{a}\right)
$$

On a catalyst segment of length $L$ we can use the following approximation

$$
\frac{\partial T_{g}}{\partial x}=\frac{T_{g, \text { out }}-T_{g, \text { in }}}{L}
$$

where $T_{g, \text { in }}$ and $T_{g, \text { out }}$ is the temperature of the gas entering and leaving the segment, respectively. Finally, by combining (45) and (46) we get

$$
\begin{array}{r}
\frac{d T_{g, \text { out }}}{d t}=-\frac{1}{(1-\varepsilon) \rho_{s} C_{p, s}}\left(v \varepsilon \rho_{g} C_{p, g} \frac{T_{g, \text { out }}-T_{g, \text { in }}}{L}\right. \\
\left.+h_{s \leftrightarrow a} a_{s \leftrightarrow a}\left(T_{g, \text { out }}-T_{a}\right)\right)
\end{array}
$$

\subsubsection{Temperature Sensors}

Since the dynamics of the temperature sensors can be quite significant models for thees are needed. The model includes conduction from the gas surrounding the sensor and radiation from the surroundings and is described below.

The density of the gas can, using the ideal gas law, be calculated as

$$
\rho=\frac{p}{R T_{g}}
$$

using this the velocity of the gas can be calculated using

$$
V=\frac{W_{\text {exh }}}{A_{\text {pipe }} \rho}
$$

The Reynolds number is calculated as

$$
R e=\frac{V D_{\text {sens }}}{\gamma}
$$

where

$$
\gamma=\frac{\mu}{\rho} .
$$

The Nusselt number is calculated according to (Holman, 1986) as
$N u=\left\{\begin{array}{rr}0.3+\frac{0.62 \operatorname{Re}^{1 / 2} \operatorname{Pr}^{1 / 3}}{\left(1+\left(\frac{0.4}{P r}\right)^{2 / 3}\right)^{1 / 4}}\left(1+\left(\frac{R e}{282000}\right)^{5 / 8}\right)^{4 / 5} \\ \frac{\operatorname{RePr} \geq 0.2}{\operatorname{RePr}<0.2}\end{array}\right.$

however this gives very low values for low mass flows and therefore a lower saturation, $N u_{\text {min }}$, is used.

The heat transfer coefficient can now be calculated as

$$
h=\frac{k_{\text {exh }} N u}{D_{\text {sens }}}
$$

By assuming that that the length of the sensor is twice the diameter of the sensor, the area of the sensor is

$$
A_{\text {sens }}=\pi D_{\text {sens }}^{2}
$$

and the mass of the sensor is

$$
m_{\text {sens }}=\rho_{\text {sens }} \frac{\pi D_{\text {sens }}^{3}}{2}
$$

The dynamics of the sensor can now be written

$$
\dot{T}_{s}=\frac{h A_{\text {sens }}\left(T_{g}-T_{s}\right)-\varepsilon \sigma A_{\text {sens }}\left(T_{s}^{4}-T_{w}^{4}\right)}{m_{\text {sens }} c_{p}}
$$

\subsubsection{Diesel Oxidation Catalyst}

The temperature of the DOC calculated as described Section 2.3.2. The oxidation of $N O$ is calculated using a map depending on the temperature of the DOC and exhaust mass flow.

\subsubsection{Diesel Particulate Filter}

The DPF is split lengthwise into five segments and each segment has a state describing its temperature. The temperature in each segment is calculated as described in Section 2.3.2, and the surface temperature is taken as the mean of the temperatures of all segments. No reactions or filtering is modeled in the DPF.

\subsubsection{Selective Catalytic Reduction Catalyst}

Like the DPF, the SCR is split lengthwise into five segments and each segment has a state describing its temperature and ammonia surface coverage. The temperature in each segment is calculated as described in Section 2.3.2. The reactions and mass balances in the catalyst is modeled similarly to (Winkler et al., 2003; Van Helden et al., 2004) and is described below.

In each segment $\mathrm{NH}_{3}$ adsorption and desorption

$$
\mathrm{NH}_{3} \longleftrightarrow \mathrm{NH}_{3}{ }^{*}
$$


are modeled using the reaction rate expressions

$$
\begin{gathered}
r_{a}=k_{a}^{0} \exp \left(\frac{-E_{a}^{0}}{R}\left(\frac{1}{T_{s}}-\frac{1}{T_{r e f}}\right)\right) C_{\mathrm{NH}_{3}}\left(1-\Theta_{\mathrm{NH}_{3}}\right) \\
r_{d}=k_{d}^{0} \exp \left(\frac{-E_{d}^{0}\left(1-\alpha \Theta_{\mathrm{NH}_{3}}\right)}{R}\left(\frac{1}{T_{s}}-\frac{1}{T_{r e f}}\right)\right) \Theta_{\mathrm{NH}_{3}}
\end{gathered}
$$

where $k_{i}$ and $E_{i}^{0}$ is the pre-exponential factor and activation energy for reaction $i$, respectively, $C_{i}$ is the concentration of specie $i, T_{s}$ is the substrate temperature, and $\Theta_{\mathrm{NH}_{3}}$ is the ammonia surface coverage.

In the SCR catalyst the following $\mathrm{NO}_{\mathrm{x}}$ reducing reactions modeled are:

$$
\begin{aligned}
4 \mathrm{NH}_{3}+4 \mathrm{NO}+\mathrm{O}_{2} & \longrightarrow 4 \mathrm{~N}_{2}+6 \mathrm{H}_{2} \mathrm{O} \\
2 \mathrm{NH}_{3}+\mathrm{NO}+\mathrm{NO}_{2} & \longrightarrow 2 \mathrm{~N}_{2}+3 \mathrm{H}_{2} \mathrm{O} \\
8 \mathrm{NH}_{3}+6 \mathrm{NO}_{2} & \longrightarrow 7 \mathrm{~N}_{2}+12 \mathrm{H}_{2} \mathrm{O}
\end{aligned}
$$

and they are modeled using the following reaction rate expressions

$$
\begin{gathered}
r_{s t d}=k_{s t d}^{0} \exp \left(\frac{-E_{s t d}}{R}\left(\frac{1}{T_{s}}-\frac{1}{T_{r e f}}\right)\right) C_{\mathrm{NO}} \Theta_{\mathrm{NH}_{3}}^{*} \\
\times\left(1-\exp \left(\frac{-\Theta_{\mathrm{NH}_{3}}}{\Theta_{\mathrm{NH}_{3}}^{*}}\right)\right) \\
r_{f s t}=k_{f s t}^{0} \exp \left(\frac{-E_{f s t}}{R}\left(\frac{1}{T_{s}}-\frac{1}{T_{r e f}}\right)\right) C_{\mathrm{NO} C_{\mathrm{NO}_{2}} \Theta_{\mathrm{NH}_{3}}} \\
r_{s l w}=k_{s l w}^{0} \exp \left(\frac{-E_{s l w}}{R}\left(\frac{1}{T_{s}}-\frac{1}{T_{r e f}}\right)\right) C_{\mathrm{NO}_{2}} \Theta_{\mathrm{NH}_{3}}
\end{gathered}
$$

$\mathrm{NH}_{3}$ oxidation

$$
4 \mathrm{NH}_{3}+3 \mathrm{O}_{2} \longrightarrow 2 \mathrm{~N}_{2}+6 \mathrm{H}_{2} \mathrm{O}
$$

is modeled by

$$
r_{o x \mathrm{NH}_{3}}=k_{o x \mathrm{NH}_{3}}^{0} \exp \left(\frac{-E_{o x \mathrm{NH}_{3}}}{R}\left(\frac{1}{T_{s}}-\frac{1}{T_{r e f}}\right)\right) \Theta_{\mathrm{NH}_{3}}
$$

Finally, hydrolysis of HNCO

$$
\mathrm{HNCO}+\mathrm{H}_{2} \mathrm{O} \longrightarrow \mathrm{NH}_{3}+\mathrm{CO}_{2}
$$

is modeled by

$$
r_{\mathrm{HNCO}}=k_{\mathrm{HNCO}}^{0} \exp \left(\frac{-E_{\mathrm{HNCO}}}{R}\left(\frac{1}{T_{s}}-\frac{1}{T_{r e f}}\right)\right) C_{\mathrm{HNCO}} .
$$

Using thees reaction rates, the surface coverage mass balance is modeled as

$$
\frac{d \Theta_{\mathrm{NH}_{3}}}{d t}=r_{a}-r_{d}-4 r_{s t d}-2 r_{f s t}-8 r_{s l w}-4 r_{o x \mathrm{NH}_{3}}
$$

and species mass balances in the gas phase are modeled by

$$
\begin{aligned}
\frac{d C_{\mathrm{HNCO}}}{d t} & =-v \frac{\partial C_{\mathrm{HNCO}}}{\partial x}-r_{\mathrm{HNCO}} \\
\frac{d C_{\mathrm{NH}_{3}}}{d t} & =-v \frac{\partial C_{\mathrm{NH}_{3}}}{\partial x}-\Omega\left(r_{a}-r_{d}\right)+r_{\mathrm{HNCO}} \\
\frac{d C_{\mathrm{NO}}}{d t} & =-v \frac{\partial C_{\mathrm{NO}}}{\partial x}-\Omega\left(4 r_{s t d}+r_{f s t}+r_{s l w}\right) \\
\frac{d C_{\mathrm{NO}_{2}}}{d t} & =-v \frac{\partial C_{\mathrm{NO}_{2}}}{\partial x}-\Omega\left(r_{f s t}+6 r_{s l w}\right)
\end{aligned}
$$

where $v$ is the velocity of the gas in the segment. All of these concentrations can be written on the form

$$
\frac{d C_{i}}{d t}=-v \frac{\partial C_{i}}{\partial x}+\sum_{j} k_{j} r_{j}
$$

with appropriate choices of $k_{j}$. By assuming the catalyst is working as a plug flow reactor, meaning there is no local accumulation of gas phase species, we get

$$
\frac{d C_{i}}{d t}=0 \Longrightarrow \frac{\partial C_{i}}{\partial x}=\frac{1}{v} \sum_{j} k_{j} r_{j}
$$

and for a segment of length $L$, given the concentration at the inlet, $C_{i, i n}$, we can calculate the concentration at the outlet, $C_{i, \text { out }}$, using the approximation

$$
C_{i, \text { out }}=C_{i, \text { in }}+\frac{L}{v} \sum_{j} k_{j} r_{j} .
$$

When using this approximation we get a singularity at $v=0$. However, this can easily be handled by limiting $v$ and not let it become to smaller than a given value. By choosing a small enough limit on $v$ the model can still produce accurate results since for small $v$ the mass flow out of the aftertreatment system is small and does not influence the results very much.

\subsubsection{Pressure Drop}

The back pressure from the EATS, $p_{\text {eats }}$, is modeled by a control volume between the turbine and the EATS using following differential equation

$$
\dot{p}_{\text {eats }}=\frac{R_{a} T_{\text {eats }}}{V_{\text {eats }}}\left(W_{t}-W_{\text {eats }}\right)
$$

where the, $W_{\text {eats }}$ is the mass flow thorough the EATS. To get $W_{\text {eats }}$ the EATS is modeled as a incompressible turbulent restriction (Eriksson and Nielsen, 2014), giving us

$$
W_{\text {eats }}= \begin{cases}C_{t u} \sqrt{\frac{p_{\text {eats }}}{R T_{\text {eats }}}} \sqrt{\Delta p}, & \Delta p \geq \Delta p_{\text {lin }} \\ C_{t u} \sqrt{\frac{p_{\text {eats }}^{R}}{R T_{\text {eats }}}} \frac{\Delta p}{\sqrt{\Delta p_{\text {lin }}}}, & \Delta p<\Delta p_{\text {lin }}\end{cases}
$$

where $\Delta p=p_{\text {eats }}-p_{a m b}$, and $\Delta p_{\text {lin }}$ is the size of the linear region and is used to make the model Lipschitz continuous. 


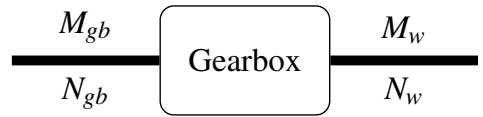

$N_{e} M_{c}^{u_{f c}} M_{e}$

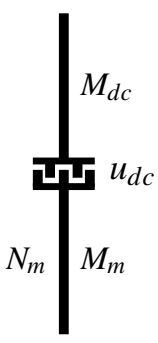

Friction Clutch

Dog Clutch

Figure 5. Components in the driveline.

\subsection{Driveline}

The driveline model consists of four components: a friction clutch, a dog clutch, a torque coupler, and a transmission. Here the equations of the components are described using the notation shown in Figure 5.

\section{Torque coupler}

The torque coupler connects the engine and the motor. To match the speed of the components a gear ratio, $i_{t c}$, is used. The equations for the torque coupler becomes

$$
\begin{aligned}
M_{t c} & =\bar{M}_{e}+i_{t c} \bar{M}_{m} \\
J_{t c} & =\bar{J}_{e}+i_{t c}^{2} \bar{J}_{m} \\
n_{e} & =n_{t c}=i_{t c} n_{m}
\end{aligned}
$$

\section{Gearbox}

The gearbox has a gear ration $i_{g}=i_{g}\left(u_{g}\right)$ that connects the speed of the torque coupler with the speed of the wheels

$$
\omega_{t c}=i_{g} \omega_{w} .
$$

The efficiency of the gearbox is also modeled using $\eta_{g b}=$ $\eta_{g b}\left(u_{g}\right)$ in the following way

$$
M_{w}=\eta_{g b}^{\operatorname{sgn}\left(M_{t c}\right)} i_{g} M_{t c} .
$$

Note that $\eta_{g b}$ depends on which gear that is engaged (typically the highest gear is more efficient than the rest of the gears).

\section{Dog clutch}

The dog clutch is used to decouple the motor from the rest of the driveline. The dog clutch can either be locked $\left(u_{d c}=1\right)$ or completely open $\left(u_{d c}=0\right)$. Mathematically this is expressed as

$$
\begin{aligned}
\bar{M}_{m} & = \begin{cases}M_{m}, & u_{d c}=1 \\
0, & u_{d c}=0\end{cases} \\
\bar{J}_{m} & = \begin{cases}J_{m}, & u_{d c}=1 \\
0, & u_{d c}=0\end{cases}
\end{aligned}
$$

\section{Friction Clutch}

The clutch model is taken from (Eriksson, 2001). The clutch position $u_{f c} \in[0,1]$ controls the friction clutch (0 means fully separated clutch and 1 means full force on the clutch discs). The clutch can either be slipping $\omega_{e} \neq \omega_{c}$ or locked $\omega_{e}=\omega_{c}$. When the clutch is slipping the two masses move independently governed by the following differential equations

$$
\begin{aligned}
J_{e} \dot{\omega}_{e} & =M_{e}-M_{c} \\
J_{t o t} \dot{\omega}_{c} & =M_{c}-M_{t o t}
\end{aligned}
$$

where $J_{t o t}$ and $M_{t o t}$ is the total inertia and torque, respectively, on the wheel side. The total inertia is the sum of the equivalent inertia of the vehicle and motor, which is

$$
J_{t o t}=\frac{I_{w}}{i_{g}^{2}}+u_{d c} i_{t c}^{2} J_{m}
$$

and the total torque is

$$
M_{t o t}=u_{d c} i_{g} M_{m}-\frac{M_{w}}{i_{g}} .
$$

In this case the torque transfered through the clutch is

$$
M_{c}=M_{\max , k} u_{c l} \operatorname{sgn}\left(w_{e}-w_{c}\right)
$$

When the clutch is locked the two systems should rotate with identical speed. The governing differential equation now becomes

$$
\left(J_{e}+J_{t o t}\right) \dot{\omega}_{e}=M_{e}-M_{t o t} .
$$

For this to hold the transfered torque through the clutch must be

$$
M_{c}=\frac{M_{e} J_{t o t}+M_{t o t} J_{e}}{J_{e}+J_{t o t}} .
$$

This torque is also compared with the maximum static torque possible to transfer through the clutch, in order to determine if the clutch should start slipping. More information about the clutch model can be found in (Eriksson, 2001).

\subsection{Chassis}

The chassis model describes the vehicles interaction with the environment by calculating the resistive forces acting on the vehicle. The total resistive force acting on the vehicle is a sum of four components

$$
F=F_{a}+F_{r}+F_{g}+F_{b}
$$

where

$$
F_{a}=\frac{1}{2} \rho C_{d} A v^{2}
$$

is the aerodynamic resistance,

$$
F_{r}=\cos \alpha m g\left(C_{r, 0}+C_{r, 1} v^{2}\right)
$$


is the rolling resistance,

$$
F_{g}=\sin \alpha m g
$$

is the gravitational force, and

$$
F_{b}=\min \left(u_{b} K_{b}, m g\right)
$$

is the force generated by the brakes. The vehicle torque is calculated as

$$
M_{\text {vehilce }}=r_{w} F \text {. }
$$

and the equivalent vehicle inertia is

$$
J_{\text {vehicle }}=m r_{w}^{2} \text {. }
$$

\section{Parameterization and Validation}

The parameterization and validation have been done using data from two vehicles, some specifications of these vehicles are shown in Table 1. The data that was used consist of measurements from a set of senors during real world driving. All models are based on physical properties of the system and as a starting point physically reasonable values on all parameters are chosen, but to get a better agreement with measurements tuning of some parameters have been done. The rest of this section contains the parameterization and validation of the different components in the model.

Table 1. Vehicle specifications

\begin{tabular}{|c|c|c|}
\hline & Vehicle 1 & Vehicle 2 \\
\hline Type & Bus & Truck \\
Engine & $5 \mathrm{Liter}$ & 10.6 Liter \\
Motor & $100 \mathrm{~kW} / 900 \mathrm{Nm}$ & $136 \mathrm{~kW} / 1050 \mathrm{Nm}$ \\
Weight & 14.5 Tonnes & 14.5 Tonnes \\
\hline
\end{tabular}

\subsection{Engine}

In the engine model the parameters in Table 2 was tuned. $V_{d}$ was taken as the actual engine size of the vehicle. The rest of the parameters were tuned using the fact that the change in mass flow is proportional to the change in engine size, i.e.

$$
W=\gamma W_{\text {org }}
$$

where

$$
\gamma=\frac{V_{d}}{V_{d, o r g}}
$$

where the index org refers to the mass flow and size of the original engine. From the model equations we also get that

$$
\begin{aligned}
R_{c} & \propto W^{1 / 3} \\
R_{t} & \propto W^{1 / 3} \\
A_{v g t, \text { max }} & \propto W
\end{aligned}
$$

and therefore the new values are taken as

$$
\begin{aligned}
R_{c} & =\gamma^{1 / 3} R_{c, \text { org }} \\
R_{t} & =\gamma^{1 / 3} R_{t, \text { org }} \\
A_{\text {vgt }, \text { max }} & =\gamma A_{\text {vgt,max }, \text { org }}
\end{aligned}
$$

When validating the engine, the measured engine speed and torque was used as inputs to the model. The rest of the control signals were held constant, $V G T$ at $60 \%$, EGR fully closed, and no engine brakes was used. From the measured engine torque the appropriate amount of fuel was calculated using the inverse of the torque model. The result from vehicle 2 is shown in Figure 6, where it can be seen that the exhaust flow from the model agree well with the measured. However, the temperature does not agree quite as well. One possible explanation for this is that relatively small errors in mass flow induce large errors in the temperature, and therefore the model can be better than what this figure gives the impression of.

Table 2. Tuned parameters in the engine model.

\begin{tabular}{|cc|}
\hline$V_{d}$ & Engine displacement \\
$R_{c}$ & Compressor radius \\
$R_{t}$ & Compressor radius \\
$A_{v g t, \max }$ & Maximal VGT area \\
\hline
\end{tabular}
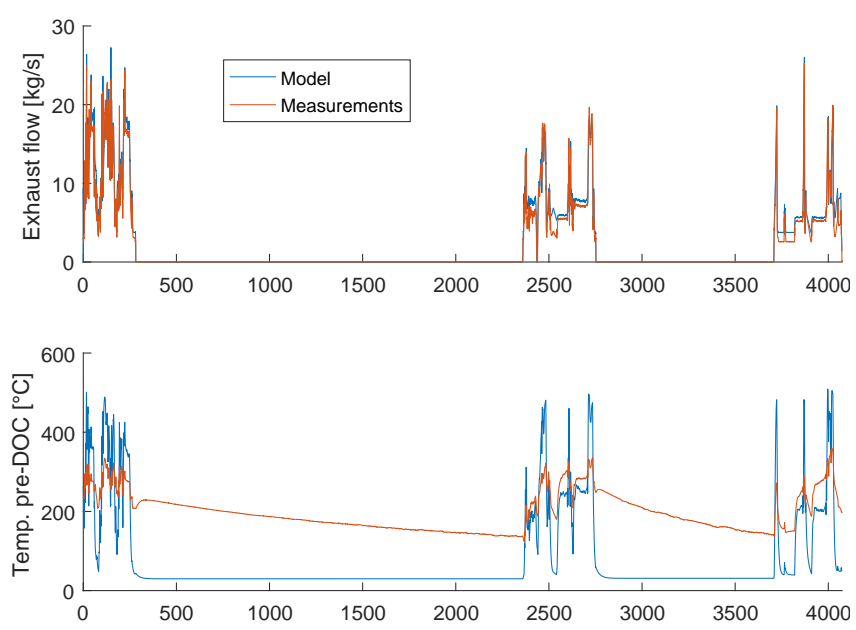

Figure 6. Measured and simulated exhaust mass flow and exhaust temperature for vehicle 2 .

\subsection{EATS}

In the EATS model the parameters in Table 3 was tuned. The lengths $L_{i}$ and diameters $D_{i}$ was scaled, compared to the original model, so that the volumes of the components scaled proportional to the change in maximal mass flow. The rest of the parameters was tuned by simulating the system using the measured mass flow and temperature before the DOC as inputs.

Since the measured temperature before the DOC was measured with a temperature sensor, the measurements 
also include the dynamics of the sensor. Therefore the measurements was first inverse filtered. By using the following model for the sensor

$$
\dot{T}_{s}=C_{s} h\left(W_{e x h}\right)\left(T_{g}-T_{s}\right)
$$

where $T_{s}$ is the temperature measured by the sensor, $T_{g}$ is the temperature of the exhaust gas, $C_{s}$ is a tuning constant, and $h$ is defined in (53). Using this we can calculate $T_{g}$ as

$$
T_{g}=T_{s}+\frac{1}{C_{s} h\left(W_{e x h}\right)} \dot{T}_{s}
$$

$\dot{T}_{S}$ was calculated numerical from the measurements and $C_{s}$ was chosen so that the time constant of the sensor was around 7 seconds at a mass flow of an idling engine. The filtered measurements and time constants for the sensor can be seen in Figure 7.

Table 3. Tuned parameters in the EATS model.

\begin{tabular}{|cc|}
\hline$L_{i}$ & Length of component $i \in\{d o c, d p f, s c r\}$ \\
$D_{i}$ & Diameter of component $i \in\{d o c, d p f, s c r\}$ \\
$\rho_{\text {solid }, i}$ & Density of component $i \in\{d o c, d p f, s c r\}$ \\
$h_{\text {solid } \leftrightarrow a m b}$ & Heat transfer coefficient, solid to ambient \\
$h_{\text {solid } \leftrightarrow \text { air }}$ & Heat transfer coefficient, solid to air \\
\hline
\end{tabular}
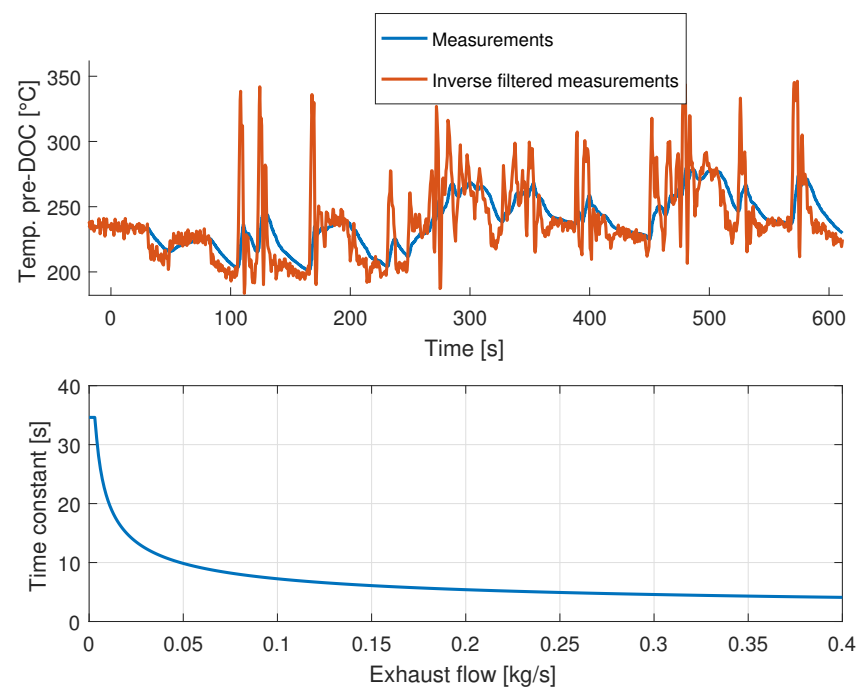

Figure 7. Inverse filtered temperature measurements and time constant for the temperature sensor.

The validation for vehicle 1 and 2 can bee seen i Figure 8 and Figure 9, respectively. As can bee seen the model and measurements agree well at higher mass flows. When the mass flow is zero, however, they do not agree well, but when the mass flow increases they quickly converge again. No validation data for the temperature after the SCR was available, but in Figure 10 measured temperature after the DPF and modeled temperature after the $\mathrm{SCR}$, for vehicle 2 , is shown. As expected the temperature after the SCR is a slightly smoothed version of the temperature after the DPF, however, fast transient due to changes in mass flow are not smoothed.
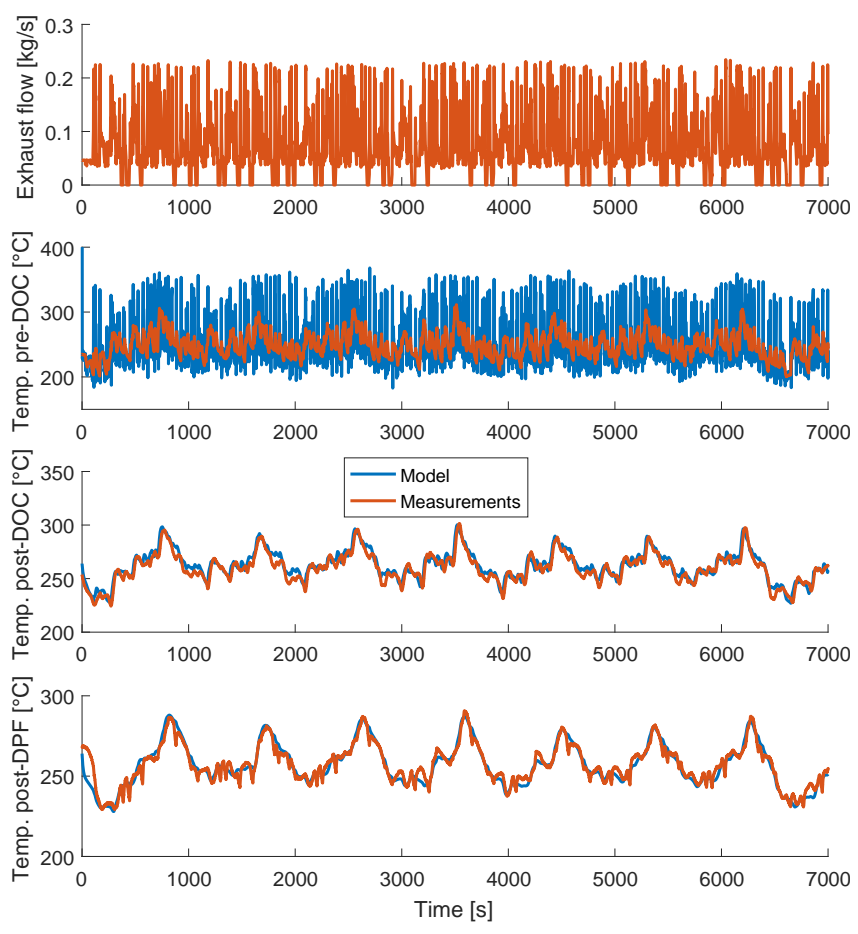

Figure 8. Exhaust flow and modeled and measured temperatures in the following places: before the DOC (here model is inverse filtered measurements), after the DOC, and after the DPF. All data is from vehicle 1 .

\subsection{Electrics}

The parameters for the motor model was estimated, using least squares, from a map of the power losses af a motor that was produced by a motor design tool developed in (Le Berr et al., 2012). The parameters for the battery was taken from (Energy, 2016).

To validate the model the required current from the motor model, when producing the same torque and at the same speed as the measured, was compared to the measured current. The measured current also include the current required from auxiliary components and therefore a current equivalent to a power of $10 \mathrm{~kW}$ was subtracted from the measured current. The result for vehicle 1 is shown in Figure 11, where it can be seen that the modeled and measured current mostly agree well, especially considering that the power required by the auxiliary components most likely varies a little with the time.

\subsection{Chassis}

The parameters for the chassis model are taken from (Eriksson et al., 2016). The validation of the chassis model was done by using the measured road slope, motor torque, engine torque, and gear as input signals to the model. The torque from the engine and motor was transformed to appropriate input signals to the engine and motor model by using the inverse of their toruqe models. Since no information of how the friction brakes were used was available a brake controller was also included. The brake controller 

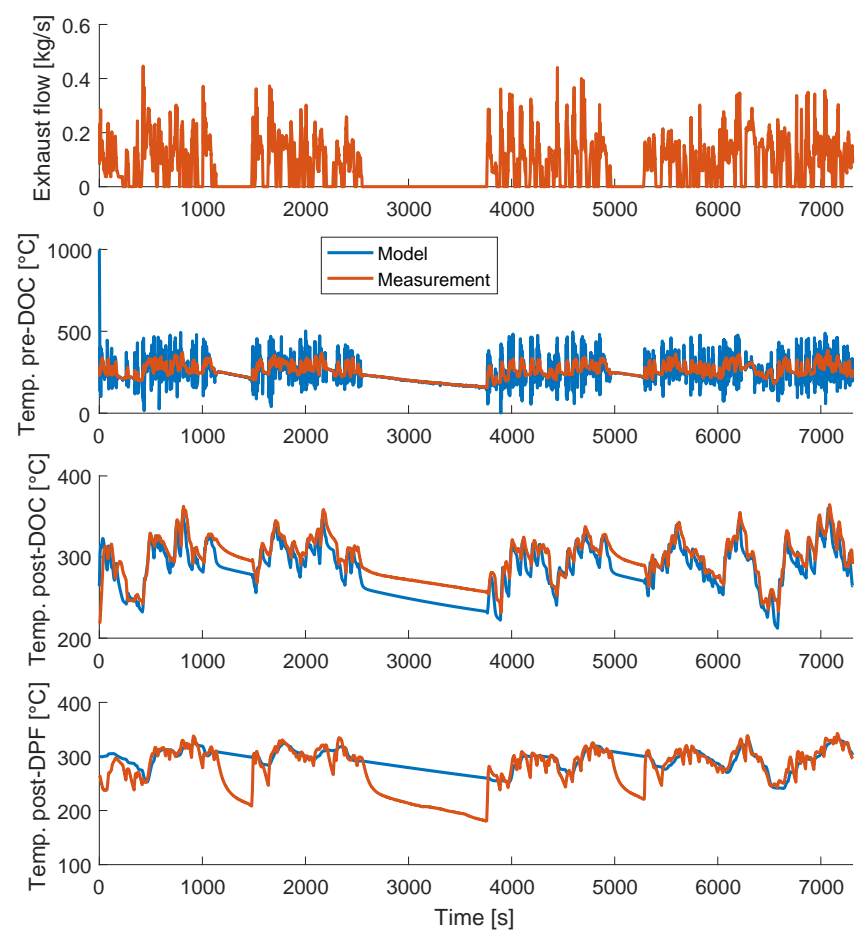

Figure 9. Exhaust flow and modeled and measured temperatures in the following places: before the DOC (here model is inverse filtered measurements), after the DOC, and after the DPF. All data is from vehicle 2 .

is a proportional controller with a dead band. The dead band is $5 \mathrm{~km} / \mathrm{h}$ and is included to avoid unnecessary braking. Also, when the measured speed was zero the brakes were applied to make sure the vehicle is standing still even if it is in a down or uphill. The result for vehicle 1 is shown in Figure 12. As can be seen the modeled and measured velocity agree well large parts of the simulation and the simulated speed is about as often higher than the measured as it is lower.

\section{Conclusions}

A model of a complete hybrid vehicle with an aftertreatment system has been developed and documented. The model contains several subcomponents and is based on the physical properties of the system. Parameterization and validation of the model have been done using measurements gathered from two vehicles during real world driving, and the model has shown to agree well with the measurements.

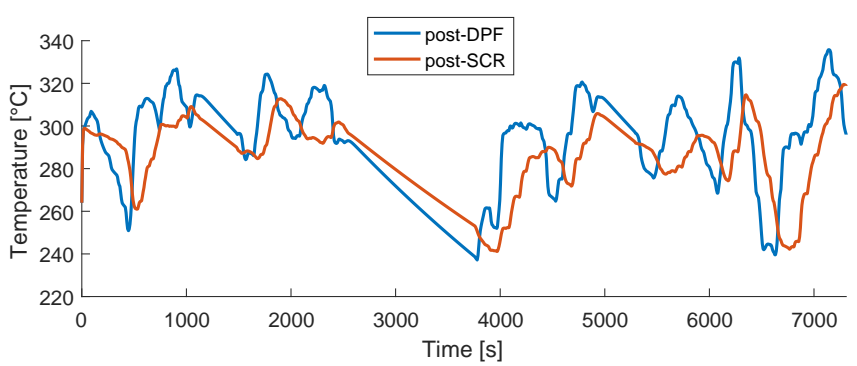

Figure 10. Measured temperature after the DPF and modeled temperature after the SCR for vehicle 2.

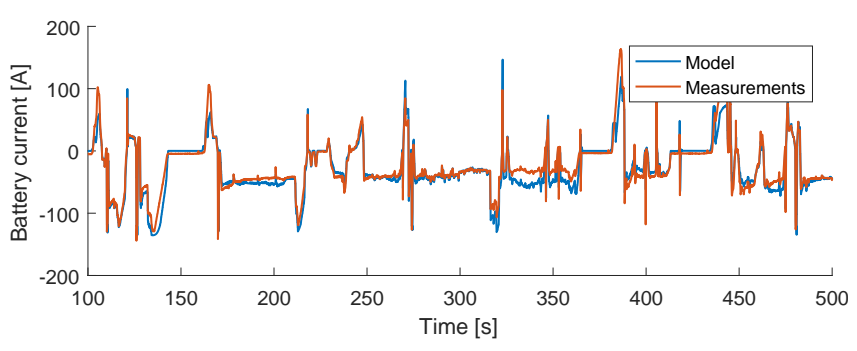

Figure 11. Measured and modeled current required by the motor for vehicle 1 . 

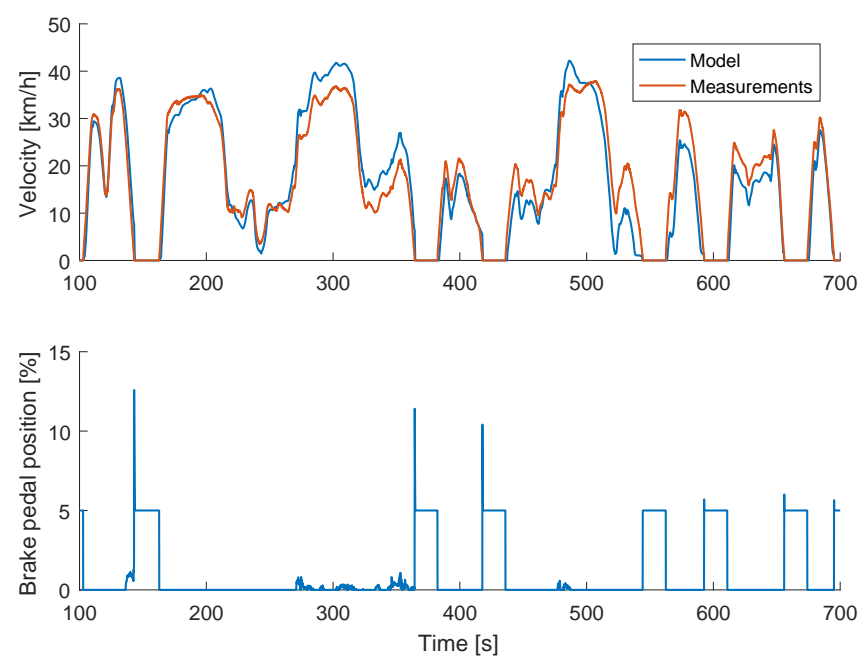

Figure 12. Measured and simulated vehicle speed or vehicle 1.

\section{References}

XALT Energy. Xalt 31 ah high power (hp) superior lithium ion cell. Available from: http://www.xaltenergy.com, [28 October 2016], 2016.

Lars Eriksson. Simulation of a vehicle in longitudinal motion with clutch lock and clutch release. Vehicular Systems, ISY Linköping Institute of Technology, Linköping, 2001.

Lars Eriksson. Mean value models for exhaust system temperatures. Technical report, SAE Technical Paper, 2002.

Lars Eriksson and Lars Nielsen. Modeling and control of engines and drivelines. John Wiley \& Sons, 2014.

Lars Eriksson, Anders Larsson, and Andreas Thomasson. The aac2016 benchmark-look-ahead control of heavy duty trucks on open roads. IFAC-PapersOnLine, 49(11), 2016.

John Heywood. Internal combustion engine fundamentals. McGraw-Hill Education, 1988.

JP Holman. Heat transfer, 1986. Mc Gran-Hill Book Company, Soythern Methodist University, 1986.

Fabrice Le Berr, Abdenour Abdelli, D-M Postariu, and R Benlamine. Design and optimization of future hybrid and electric propulsion systems: An advanced tool integrated in a complete workflow to study electric devices. Oil \& Gas Science and Technology-Revue dâĂŹIFP Energies nouvelles, 67(4): 547-562, 2012.

Christofer Sundström, Erik Frisk, and Lars Nielsen. A new electric machine model and its relevance for vehicle level diagnosis. International Journal of Modeling, Identification and Control (IJMIC), 24(1):1-9, 2015.

Rinie Van Helden, Ruud Verbeek, Frank Willems, and Reinier van der Welle. Optimization of urea scr denox systems for hd diesel engines. Technical report, SAE Technical Paper, 2004.
Johan Wahlström and Lars Eriksson. Modelling diesel engines with a variable-geometry turbocharger and exhaust gas recirculation by optimization of model parameters for capturing non-linear system dynamics. Proceedings of the Institution of Mechanical Engineers, Part D: Journal of Automobile Engineering, 225(7):960-986, 2011.

Frank Willems and Darren Foster. Integrated powertrain control to meet future co 2 and euro- 6 emissions targets for a diesel hybrid with scr-deno x system. In 2009 American Control Conference, pages 3944-3949. IEEE, 2009.

Christian Winkler, Peter Flörchinger, MD Patil, Jürgen Gieshoff, Paul Spurk, and Marcus Pfeifer. Modeling of scr denox catalyst-looking at the impact of substrate attributes. Technical report, SAE Technical Paper, 2003.

\section{A Notation and Subscripts}

\section{Notation}

$\gamma \quad$ Specific heat capacity ratio (-)

$\varepsilon \quad$ Emissivity (-)

$\eta \quad$ Efficiency (-)

$\Theta \quad$ Surface coverage (-)

$\rho \quad$ Density $\left(\mathrm{kg} / \mathrm{m}^{3}\right)$

$\sigma \quad$ Stefan-Boltzmann constant $\left(\mathrm{W} / \mathrm{m}^{2} \mathrm{~K}^{4}\right)$

$\omega \quad$ Rotational speed $(\mathrm{rad} / \mathrm{s})$

A Area $\left(\mathrm{m}^{2}\right)$

$c_{p} \quad$ Const. pressure specific heat capacity $(\mathrm{J} / \mathrm{kgK})$

$D \quad$ Diameter (m)

$E^{0} \quad$ Activation energy (J)

$F_{v} \quad$ Gray body view factor (-)

$g \quad$ Acceleration due to gravity $\left(\mathrm{m} / \mathrm{s}^{2}\right)$

$h \quad$ Heat transfer coefficient $\left(\mathrm{W} / \mathrm{m}^{2} \mathrm{~K}\right)$

I Current (A)

$i \quad$ Gear ratio (-)

$J \quad$ Inertia $\left(\mathrm{kgm}^{2}\right)$

$k^{0} \quad$ Pre-exponential factor (-)

$M \quad$ Torque $(\mathrm{Nm})$

$m \quad$ Mass $(\mathrm{kg})$

$N \quad$ Rotational speed (r/min)

$\mathrm{Nu} \quad$ Nusselt number (-)

$P \quad$ Power (W)

$p \quad$ Pressure $(\mathrm{Pa})$

$Q \quad$ Heat $(\mathrm{J})$

$q_{H V} \quad$ Heating value of fuel $(\mathrm{J} / \mathrm{kg})$

$R \quad$ Gas constant $(\mathrm{J} / \mathrm{kgK})$

$R \quad$ Resistance $(\Omega)$

Re Reynolds number (-)

$T$ Temperature (K)

$U \quad$ Voltage (V)

$u \quad$ Control signal

$V \quad$ Volume $\left(\mathrm{m}^{3}\right)$

$v \quad$ Velocity $(\mathrm{m} / \mathrm{s})$

$W \quad$ Mass flow $(\mathrm{kg} / \mathrm{s})$

$X \quad$ Mass fraction (-) 


\section{Subscripts}

\begin{tabular}{ll} 
a & Air \\
aft.turb & After turbo \\
amb & Ambient \\
b & Battery \\
bpv & Back pressure valve \\
c & Compressor \\
crb & Compressing release brake \\
cv & Convection \\
cyl & Cylinder \\
d & Displacement \\
dc & Dog clutch \\
$\mathrm{e}$ & Internal combustion engine \\
eats & Engine after treatment system \\
egr & Exhaust gas recirculation \\
ei & Engine in \\
em & Exhaust manifold \\
eo & Engine out \\
exh & Exhaust \\
$\mathrm{f}$ & Fuel \\
fc & Friction clutch \\
fric & Friction \\
gb & Gearbox \\
$\mathrm{g}$ & Gas \\
$\mathrm{i}$ & Internal \\
$\mathrm{ig}$ & Indicated gross \\
igch & Ignition chamber \\
im & Intake manifold \\
$\mathrm{m}$ & Electric machine \\
$\mathrm{O}$ & Oxygen \\
oc & Open circuit \\
$\mathrm{p}$ & Pump \\
$\mathrm{t}$ & Turbine \\
us & Upstream \\
vgt & Variable geometry turbine \\
vol & Volume \\
$\mathrm{w}$ & Wall \\
& \\
\hline
\end{tabular}

